\title{
HARDY-LITTLEWOOD-PÓLYA INEQUALITIES AND HAUSDORFF OPERATORS ON BLOCK SPACES
}

\author{
KWOK-PUN Ho
}

Abstract. We establish the Hardy-Littlewood-Pólya inequality, the Hardy inequality and the Hilbert inequality on block spaces. Furthermore, we also have the boundedness of the Hausdorff operators on block spaces.

Mathematics subject classification (2010): 26D10, 26D15, 42B35, 46E30.

Keywords and phrases: Hardy-Littlewood-Pólya inequality, Hausdorff operator, block space, Morrey space.

\section{REFERENCES}

[1] D. Adams And J. XiaO, Morrey spaces in harmonic analysis, Ark. Mat., 50, (2012), 201-230.

[2] K. ANDERSEN, Boundedness of Hausdorff operators on $L^{p}\left(\mathbb{R}^{n}\right), H^{1}\left(\mathbb{R}^{n}\right)$, and $B M O\left(\mathbb{R}^{n}\right)$, Acta. Sci. Math. (Szeged), 69, (2003), 409-418.

[3] J. AlvareZ, Continuity of Calderón-Zygmund type operators on the predual of a Morrey space, Proc. of Clifford Algebras in Analysis, Studies in Advanced Math., CRC Press.

[4] O. Blasco, A. Ruiz And L. Vega, Non interpolation in Morrey-Campanato and Block spaces, Ann. Scuola Norm. Sup. Pisa Cl. Sci (4), 28, (1999), 31-40.

[5] G. BROWn AND F. MóRICZ, The Hausdorff operator and the quasi Hausdorff operator on the space $L^{p}, 1 \leqslant p<\infty$, Math. Inequal. Appl., 3, (2000), 105-115.

[6] G. BRown AND F. MóRICZ, Multivariate Hausdorff operators on the spaces $L^{p}\left(\mathbb{R}^{n}\right)$, J. Math. Anal. Appl., 271, (2002), 443-454.

[7] V. BuRENKOV, Recent progress in the problem of the boundedness of classical operators of real analysis in general Morrey-type spaces. I, Eurasian Math. J., 3, (2012), 11-32.

[8] V. BURENKOV, Recent progress in the problem of the boundedness of classical operators of real analysis in general Morrey-type spaces. II, Eurasian Math. J., 4, (2013), 21-45.

[9] J. C. CHEN, D. S. FAN AND J. Li, Hausdorff operators on function spaces, Chin. Ann. Math. Ser. B, 33, (2012), 537-556.

[10] J. C. Chen, D. S. FAn And C. J. Zhang, Multilinear Hausdorff operators and their best constants, Acta Math. Sin. Ser. B, 28, (2012), 1521-1530.

[11] J. C. CHEN, D. S. FAN AND S. L. WANG, Hausdorff operators on Euclidean spaces, Appl. Math. J. Chinese Univ., 28, (2013), 548-564.

[12] K. L. CheUnG AND K.-P. Ho, Boundedness of Hardy-Littlewood maximal operator on block spaces with variable exponent, Czechoslovak Math. J., 139, (2014), 159-171.

[13] D. Edmunds And W. Evans, Hardy Operators, Function Spaces and Embeddings, Springer, Berlin Heidelberg New York, 2004.

[14] G. Folland, Real Analysis: Modern Techniques and Their Applications, John Wiley \& Sons, U.S.A., 1984.

[15] G. GaO And Y. Zhong, Some inequalities for Hausdorff operators, Math. Inequal. Appl., 17, (2014), 1061-1078.

[16] C. GeORgakis, The Hausdorff mean of a Fourier-Stieltjes transform, Proc. Amer. Math. Soc., 116, (1992), 465-471.

[17] D. Giang And F. MóRICZ, The Cesáro operator is bounded on the Hardy space $H^{1}$, Acta Sci. Math. (Szeged), 61, (1995), 535-544. 
[18] D. GiAng AND F. MóRICZ, The two dimensional Cesáro operator is bounded on the multi-parameter Hardy space $H^{1}(\mathbb{R} \times \mathbb{R})$, Acta Sci. Math. (Szeged), 63, (1997), 281-290.

[19] F. HAUSDORFF, Summationmethoden und Momentfolgen I, Math. Z., 9, (1921), 74-109.

[20] G. Hardy, J. Littlewood and G. Pólya, Inequalities, Cambridge University Press, New York, 1988.

[21] K.-P. Ho, Sobolev-Jawerth embedding of Triebel-Lizorkin-Morrey-Lorentz spaces and fractional integral operator on Hardy type spaces, Math. Nachr., 287, (2014), 1674-1686.

[22] K.-P. Ho, Atomic decomposition of Hardy-Morrey spaces with variable exponents, Ann. Acad. Sci. Fenn. Math., 40, (2015), 31-62.

[23] K.-P. Ho, Vector-valued operators with singular kernel and Triebel-Lizorkin-block spaces with variable exponents, (accepted by Kyoto J. Math.).

[24] K.-P. Ho, Hardy's inequality and Hausdorff operators on rearrangement-invariant Morrey spaces, (accepted by Publ. Math. (Debrecen)).

[25] E. Kalita, Dual Morrey spaces, Dokl. Math., 58, (1998), 85-87.

[26] Y. KAnJin, The Hausdorff operators on the real Hardy spaces $H^{p}(\mathbb{R})$, Studia Math., 148, (2001), $37-45$.

[27] A. LeRner AND E. LiflyAnd, Multidimensional Hausdorff operators on the real Hardy spaces, J. Aust. Math. Soc., 83, (2007), 79-86.

[28] E. LiflyAnd, Boundedness of multidimensional Hausdorff operators on $H^{1}\left(\mathbb{R}^{n}\right)$, Acta Sci. Math. (Szeged), 74, (2008), 845-851.

[29] E. LiflyAND, Hausdorff operators on Hardy spaces, Eurasian Math. J., 4, (2013), 101-141.

[30] E. LIFL YAND AND F. MóRICZ, The Hausdorff operators is bounded on the real Hardy space $H^{1}\left(\mathbb{R}^{n}\right)$, Proc. Amer. Math. Soc., 128, (2000), 1391-1396.

[31] E. LIFLYAND AND F. MóRICZ, The multi-parameter Hausdorff operator is bounded on the product Hardy space $H^{11}(\mathbb{R} \times \mathbb{R})$, Analysis (Munich), 21, (2001), 107-118.

[32] E. LiflYAND AND A. MiYACHI, Boundedness of the Hausdorff operators in $H^{p}$ spaces, $0<p<1$, Studia Math., 194, (2009), 279-292.

[33] Y. Meyer, M. Taibleson And G. Weiss, Some functional analytic properties of the space $B_{q}$ generated by blocks, Indiana Univ. Math. J., 34, (1985), 493-515.

[34] A. MiYACHI, Boundedness of the Cesáro operator in Hardy spaces, J. Fourier Anal. Appl., 10, (2004), $83-92$.

[35] F. MóRICZ, Multivariate Hausdorff operators on the spaces $H^{p}\left(\mathbb{R}^{n}\right)$ and $B M O\left(\mathbb{R}^{n}\right)$, Anal. Math., 31, (2005), 31-41.

[36] C. Morrey, On the solutions of quasi-linear elliptic partial differential equations, Trans. Amer. Math. Soc., 43, (1938), 126-166.

[37] B. Opic And A. Kufner, Hardy-type inequalities, Pitman Reserach Notes in Math. Series 219, Longman Sci. and Tech, Harlow, 1990.

[38] A. SiskAKIS, The Cesáro operator is bounded on $H^{1}$, Proc. Amer. Math. Soc., 110, (1990), 461-462.

[39] F. SORIA, Characterizations of classes of functions generated by blocks and associated Hardy spaces, Indiana Univ. Math. J., 34, (1985), 463-492.

[40] F. WEISZ, The boundedness of the Hausdorff operator on multi-dimensional Hardy spaces, Analysis (Munich), 24, (2004), 183-195.

[41] C. Zorko, Morrey Space, Proc. Amer. Math. Soc., 98, (1986), 586-592. 